\title{
Enhanced capital-asset pricing model for the reconstruction of bipartite financial networks
}

\author{
Tiziano Squartini, ${ }^{1}$ Assaf Almog, ${ }^{2,3}$ Guido Caldarelli, ${ }^{1,4,5}$ Iman van Lelyveld, ${ }^{6,7}$ Diego Garlaschelli, ${ }^{2}$ and Giulio Cimini ${ }^{1,4, *}$ \\ ${ }^{1}$ IMT School for Advanced Studies, Piazza San Francesco 19, 55100 Lucca, Italy \\ ${ }^{2}$ Instituut-Lorentz, for Theoretical Physics, Leiden Institute of Physics, University of Leiden, Niels Bohrweg 2, \\ 2333 CA Leiden, The Netherlands \\ ${ }^{3}$ Department of Industrial Engineering, Tel-Aviv University, Ramat Aviv 699780, Israel \\ ${ }^{4}$ Istituto dei Sistemi Complessi, CNR UoS Università “Sapienza,” Piazzale Aldo Moro 5, 00185 Rome, Italy \\ ${ }^{5}$ European Centre for living technology, Università di Venezia "Ca' Foscari”, S. Marco 2940, 30124 Venice, Italy \\ ${ }^{6}$ De Nederlandsche Bank, P.O. Box 98, 1000 AB Amsterdam, The Netherlands \\ ${ }^{7}$ VU University, De Boelelaan 1105, 1081 HV Amsterdam, The Netherlands
}

(Received 26 April 2017; revised manuscript received 7 September 2017; published 27 September 2017)

\begin{abstract}
Reconstructing patterns of interconnections from partial information is one of the most important issues in the statistical physics of complex networks. A paramount example is provided by financial networks. In fact, the spreading and amplification of financial distress in capital markets are strongly affected by the interconnections among financial institutions. Yet, while the aggregate balance sheets of institutions are publicly disclosed, information on single positions is mostly confidential and, as such, unavailable. Standard approaches to reconstruct the network of financial interconnection produce unrealistically dense topologies, leading to a biased estimation of systemic risk. Moreover, reconstruction techniques are generally designed for monopartite networks of bilateral exposures between financial institutions, thus failing in reproducing bipartite networks of security holdings (e.g., investment portfolios). Here we propose a reconstruction method based on constrained entropy maximization, tailored for bipartite financial networks. Such a procedure enhances the traditional capital-asset pricing model (CAPM) and allows us to reproduce the correct topology of the network. We test this enhanced CAPM (ECAPM) method on a dataset, collected by the European Central Bank, of detailed security holdings of European institutional sectors over a period of six years (2009-2015). Our approach outperforms the traditional CAPM and the recently proposed maximum-entropy CAPM both in reproducing the network topology and in estimating systemic risk due to fire sales spillovers. In general, ECAPM can be applied to the whole class of weighted bipartite networks described by the fitness model.
\end{abstract}

DOI: 10.1103/PhysRevE.96.032315

\section{INTRODUCTION}

The recent financial crises have highlighted the importance of correctly evaluating systemic risk in financial markets, by explicitly considering the role played by financial interconnections (both as direct exposures through bilateral contracts and indirect exposures through common assets holding [1-6]) in the spreading of financial distress [7-11]. Thus, characterizing the underlying network structure of financial systems has become not only a scientific but also an institutional priority [12-16]. However, while financial institutions have to disclose their aggregated exposures (eventually split into major categories), detailed data on their single positions are generally confidential and, thus, unaccessible. For this reason, reconstructing the network structure from the available data has recently attracted much attention [17-29]. Yet, while several efforts have been devoted so far to reconstruct monopartite networks of bilateral exposures between financial institutions, methods to reconstruct the bipartite structure of assets ownership by such institutions are still little explored [30]. Assessing the portfolio composition of both financial actors and the institutional sectors they belong to is, however, crucial in terms of systemic stability. In fact, portfolio overlaps have the potential to trigger fire sales, i.e., downward spirals for illiquid asset prices due to self-reinforcing sell orders [31-35]. Fire sales spillovers are also dangerous because they create

*Corresponding author: giulio.cimini@imtlucca.it incentives to hoard liquidity, thus activating a spiral potentially leading to a complete freeze of the financial system [36-41].

Reconstruction of monopartite networks of direct exposures between financial institutions (such as interbank markets) is typically pursued through dense maximum-entropy techniques that use the available information as constraints to be satisfied $[17,18]$. The major drawback of these approaches is, however, that of generating unrealistically (almost) fully connected topologies which, in turn, were shown to underestimate systemic losses due to counterparty risk $[19,20]$. Several techniques have thus been developed to obtain sparse reconstructions [20,22-24] by inferring connection probabilities via the aggregated exposures [25]. While these approaches either explore the whole range of possible network densities or directly make use of ad hoc density values, a recently proposed bootstrapping algorithm allows one to obtain an accurate guess of the true network density relying on small, informative, subsets of nodes $[42,43]$. The latter approach has then been developed into a statistically grounded reconstruction procedure, which assesses connection probabilities through a configuration model (CM) [44,45] tailored on the estimated density and induced by the aggregated exposures used as fitnesses [46,47]. In order to evaluate the weights of connections, the approach can be complemented either with a degree-corrected gravity model (dcGM) [27] or with an enhanced CM (ECM [48]) [28]. As pointed out by recent studies [49,50], this method outperforms other existing (probabilistic) reconstruction techniques. 
Differently from interbank markets, networks of institutional portfolio holdings are systems composed by two classes of nodes: institutions (or sectors) and financial instruments. In this case a reconstruction method has to account for the bipartite structure of the system, since connections are allowed only between nodes belonging to different classes. Standard maximum entropy techniques have been extended also to deal with such systems, generating candidate configurations according to the capital-asset pricing model (CAPM) - for which investors choose their portfolio so that each position on a given asset is proportional to that asset's market capitalization [51,52]. Recently, a cross-entropy maximization technique constraining weight expectations to match CAPM values [maximum-entropy CAPM (MECAPM)] has also been formalized [30]. Yet, both approaches predict very dense configurations, thus failing in reproducing the real network structure, and eventually underestimating systemic risk arising from fire sales spillovers [32] (although this may depend on the metric chosen [30]).

In this paper we aim at overcoming the density issue for bipartite networks reconstruction, by defining an enhanced CAPM (ECAPM), which extends the fitness-induced CM introduced for monopartite networks [27] to the bipartite case. In a nutshell, the method consists of a two-step inference procedure: first, links presence is assessed through a bipartite $\mathrm{CM}$ (BiCM) [53] calibrated using the aggregated balance sheet data as fitnesses; then, weights are estimated via the degree-corrected CAPM. To validate our method, we use a unique dataset of security holdings by institutional sectors in Europe (in particular, we have the detailed exposures of long-term security bonds between sectors). Since we have full information on these data (which is confidential and not publicly available), we are able to precisely assess the accuracy of our reconstruction method.

\section{METHOD}

\section{A. Notation}

We start by introducing the notation that is used throughout the paper. Assets ownership data are represented as a weighted, undirected, bipartite network $G_{0}$ of $N$ investors portfolios and $M$ assets. The generic element of the $N \times M$ biadjacency matrix, $w_{i \alpha}$, is the value of asset $\alpha$ held by portfolio $i$. The aggregated balance sheet information is thus given by the nodes strengths, namely, the market value of portfolio $i, V_{i}=\sum_{\alpha} w_{i \alpha}$, and the market capitalization of asset $\alpha$, $C_{\alpha}=\sum_{i} w_{i \alpha}$. Connection patters are instead described by the $N \times M$ binary biadjacency matrix, the generic element $a_{i \alpha}$ of which equals 1 if $w_{i \alpha}>0$ and zero otherwise. Nodes degrees are then given by the number of different assets held by each portfolio $i, k_{i}=\sum_{\alpha} a_{i \alpha}$, and by the number of investors in each asset $\alpha, d_{\alpha}=\sum_{i} a_{i \alpha} . W=\sum_{i \alpha} w_{i \alpha}$ denotes the total economic value of the system, and $L=\sum_{i \alpha} a_{i \alpha}$ is the total number of connections (ownership relations) in the network.

\section{B. Problem statement}

Suppose we have limited information on $G_{0}$ : we know the whole strength sequences $\left\{V_{i}\right\}_{i=1}^{N}$ and $\left\{C_{\alpha}\right\}_{\alpha=1}^{M}$ and the total number of links $L$ (note that strengths are usually publicly available and, thus, easy to access, whereas $L$ is more difficult to obtain and is typically proxied through density sampling or bootstrapping techniques [42,54]). In this situation, our goal is to find the optimal estimate for $X\left(G_{0}\right)$, the value of a generic property $X$ measured on the real network $G_{0}$ on the basis of the information available.

The ECAPM network reconstruction procedure works in two main steps: it supposes $G_{0}$ as drawn from an appropriate ensemble $\Omega$ of bipartite graphs, defined by a fitness-induced $\mathrm{BiCM}$ for links presence and by a degree-corrected CAPM for links weight. In what follows, we will label with the tilde symbol $\sim$ quantities of networks drawn from $\Omega$.

\section{Topology reconstruction}

If we knew the degree of each node in the network, we could use the BiCM [53] to directly generate $\Omega$ as the ensemble of binary, undirected bipartite networks satisfying the degree constraints $\left\langle k_{i}\right\rangle_{\Omega}=k_{i}\left(G_{0}\right), i=1 \ldots N$ and $\left\langle d_{\alpha}\right\rangle_{\Omega}=d_{\alpha}\left(G_{0}\right)$, $\alpha=1 \ldots M$. This would imply a probability distribution over $\Omega$ defined by a set of Lagrange multipliers $\left\{x_{i}\right\}_{i=1}^{N}$ and $\left\{y_{\alpha}\right\}_{\alpha=1}^{M}$ (one for each node) associated to the degrees, so that the connection probability between nodes $i$ and $\alpha$ is

$$
p_{i \alpha} \equiv\left\langle\tilde{a}_{i \alpha}\right\rangle_{\Omega}=\frac{x_{i} y_{\alpha}}{1+x_{i} y_{\alpha}},
$$

independently for all nodes pairs. Here, however, we are studying the case where node degrees are unknown, yet we know the total number $L$ of links. Thus, while we cannot directly use the BiCM, we can resort to the fitness ansatz, which assumes the network topology to be determined by intrinsic node properties named fitnesses. This approach has been successfully used in the past to model several economic and financial networks, by assuming a proportionality between fitnesses and Lagrange multipliers $[47,55,56]$. Thus, as in the case of monopartite financial networks [27,28], we assume the strengths (which we know) to represent node-specific fitnesses and be linearly proportional to the BiCM Lagrange multipliers (induced by degrees): $x_{i} \equiv \sqrt{z_{V}} V_{i}, \forall i$ and $y_{\alpha} \equiv \sqrt{z_{C}} C_{\alpha}, \forall \alpha$. Thanks to this assumption, our task reduces to determining only one proportionality constant, which is obtained by equating the ensemble average of the total number of links with the (known) total number $L$ of links of $G_{0}$ :

$$
\langle L\rangle_{\Omega} \equiv \sum_{i} \sum_{\alpha} \frac{z V_{i} C_{\alpha}}{1+z V_{i} C_{\alpha}}=L\left(G_{0}\right)
$$

where we have defined $z=\sqrt{z_{V} z_{C}}$. Equation (2) is an algebraic equation in $z$ with a single positive solution, which is then used to estimate the linking probabilities of Eq. (1):

$$
p_{i \alpha} \equiv\left\langle\tilde{a}_{i \alpha}\right\rangle_{\Omega}=\frac{z V_{i} C_{\alpha}}{1+z V_{i} C_{\alpha}} \quad \forall(i, \alpha) .
$$

By preserving the network density, the topology predicted by Eq. (3) generally differs substantially from the one characterizing the fully, or very densely, connected configurations predicted by the CAPM and MECAPM, respectively.

\section{Weights reconstruction}

If the information on nodes degrees were accessible, the formal approach to obtain a weighted reconstructed network 
would prescribe to use the ECM to maximize entropy, constraining both degrees and strengths. The observed degree sequence might be in principle replaced by its estimate from the first step of the method [28], yet this procedure becomes unfeasible for large networks, since it requires the solution of $2(N+M)$ coupled nonlinear equations. The alternative approach that we follow here is to make use of the CAPM framework, which can be seen as the bipartite counterpart of the standard gravity model used for monopartite networks $[17,19]$. In its original version, CAPM assigns weights as

$$
\tilde{\omega}_{i \alpha}=V_{i} C_{\alpha} / W \text {. }
$$

As a result, the model reproduces the original strengths sequence only when fully connected networks are considered. To account for a network topology determined by nontrivial connection probabilities, we can extend the dcGM prescription proposed in [27] to bipartite networks. Weights of individual links are thus determined as

$$
\tilde{w}_{i \alpha}=\frac{V_{i} C_{\alpha}}{W p_{i \alpha}} \tilde{a}_{i \alpha}=\left(z^{-1}+V_{i} C_{\alpha}\right) \frac{\tilde{a}_{i \alpha}}{W} .
$$

Notably, this equation ensures that the expected values of the reconstructed weights coincide with the CAPM ones, $\left\langle\tilde{w}_{i \alpha}\right\rangle_{\Omega} \equiv \tilde{\omega}_{i \alpha}, \forall(i, \alpha)$, thus preserving the strength sequence on average, irrespective of the underlying reconstructed topology [28].

\section{E. Remarks}

To sum up, the set of connection probabilities defined in Eq. (3) and of link weights defined in Eq. (5) univocally determine the ensemble $\Omega$, so that the value of the generic quantity $X$ in $G_{0}$ can be estimated as its ensemble average $\langle X\rangle_{\Omega}$. In the following, we will show results of the ECAPM reconstruction procedure for a case study of security holdings by European institutional sectors, which we believe can be of interest to both researchers and practitioners. A more exhaustive analysis of ECAPM can be found in the Appendix, where we show the analytic derivation of the model in the sparse network limit, as well as a more general formulation using the continuous approximation of the fitness model (in the latter case, we show how to write the expected values of the quantities of interest in terms of moments of the involved probability distributions).

Before moving further, let us explain the difference between the ECAPM and the MECAPM [30] reconstruction techniques (see the Appendix for further details). Although both approaches predict average link weights matching the CAPM values, MECAPM leads to connection probabilities of the form $q_{i \alpha}=\tilde{\omega}_{i \alpha} /\left(1+\tilde{\omega}_{i \alpha}\right)$, which is the special case $z=1 / W$ of Eq. (3). These probabilities then assume values very close to unity even for moderately large weights. This is due to the very definition of MECAPM, obtained by maximizing entropy constraining only the strength sequence (i.e., without accounting for the network connectivity), a prescription which redistributes weights as evenly as possible- thus generating very dense networks.

\section{DATA}

The dataset we use to test our reconstruction method is based on the Security Holding Statistics (SHS) collected by the
TABLE I. Institutional sector classification by the European Central Bank.

\begin{tabular}{ll}
\hline \hline Sector & Description \\
\hline s_11 & Nonfinancial corporations \\
s_121 & The central bank \\
s_122 & Deposit-taking corporations except the central bank \\
s_123 & Money market funds (MMFs) \\
s_124 & Non-MMF investment funds \\
s_125 & Other financial intermediaries, except ICs and PFs \\
s_126 & Financial auxiliaries \\
s_127 & Captive financial institutions and money lenders \\
s_128 & Insurance corporations (ICs) \\
s_129 & Pension funds (PFs) \\
s_13 & General government \\
s_14 & Households (only in holders) \\
s_15 & Nonprofit institutions serving households \\
s_X & Not allocated/unspecified \\
\hline \hline
\end{tabular}

European Central Bank. The data have quarterly information on each individual security at the country-sector level, covering a time span from $2009 Q 1$ to $2015 Q 2$, and is reported at the current Euro value. Holding data include four types of financial tools: long-term security bonds, short-term security bonds, money market funds, and equity. A distinctive feature of the dataset is that the holding information is collected for each international securities identification number (ISIN).

To carry out our analysis, we focus on long-term security bonds and consider quarterly snapshots of the debt exposure of 14 different institutional sectors, with standard European Central Bank classification (see Table I), for each of the $19 \mathrm{Eu}-$ ropean reporting countries. This results in a first layer of $N=$ 266 holders nodes, each corresponding to a specific countrysector combination. The second layer represents the issuers of the securities, where we aggregated the specific ISINs to the same country-sector classification, with the exception of the households (only 13 sectors). Since the reporting countries are required to report on any equity, with no limitation on the location of security issuer, the second layer is composed of all the possible issuers nodes from 242 countries (including the 19 reporting countries) resulting in $M=3146$ country-sector combinations. Each link $w_{i \alpha}$ thus corresponds to the holdings of holder $i$ (aggregated to the country sector) of the securities issued by issuer $j$ (aggregated to the country sector).

Overall, we have 24 temporal snapshots (quarters) of the data, bearing the complex networks signature of broad degree and strength distributions (Fig. 1). Notice that dense reconstruction methods such as CAPM and MECAPM would not be able to replicate these heterogeneous topological features.

We remark that the magnitude of the collected holdings is substantial: for instance, total holdings by countries belonging to the Euro area amount to around 18.3 trillion Euro at the end of June 2014, thus representing a relevant share of the total financial system. While the information on SHS marginals (e.g., the total asset of a particular holder sector) is relatively easy to access, the full availability of the SHS provides us with a unique opportunity to test the effectiveness of our method in reconstructing the network structure of such an important and large-scale financial system. For the sake of confidentiality, 

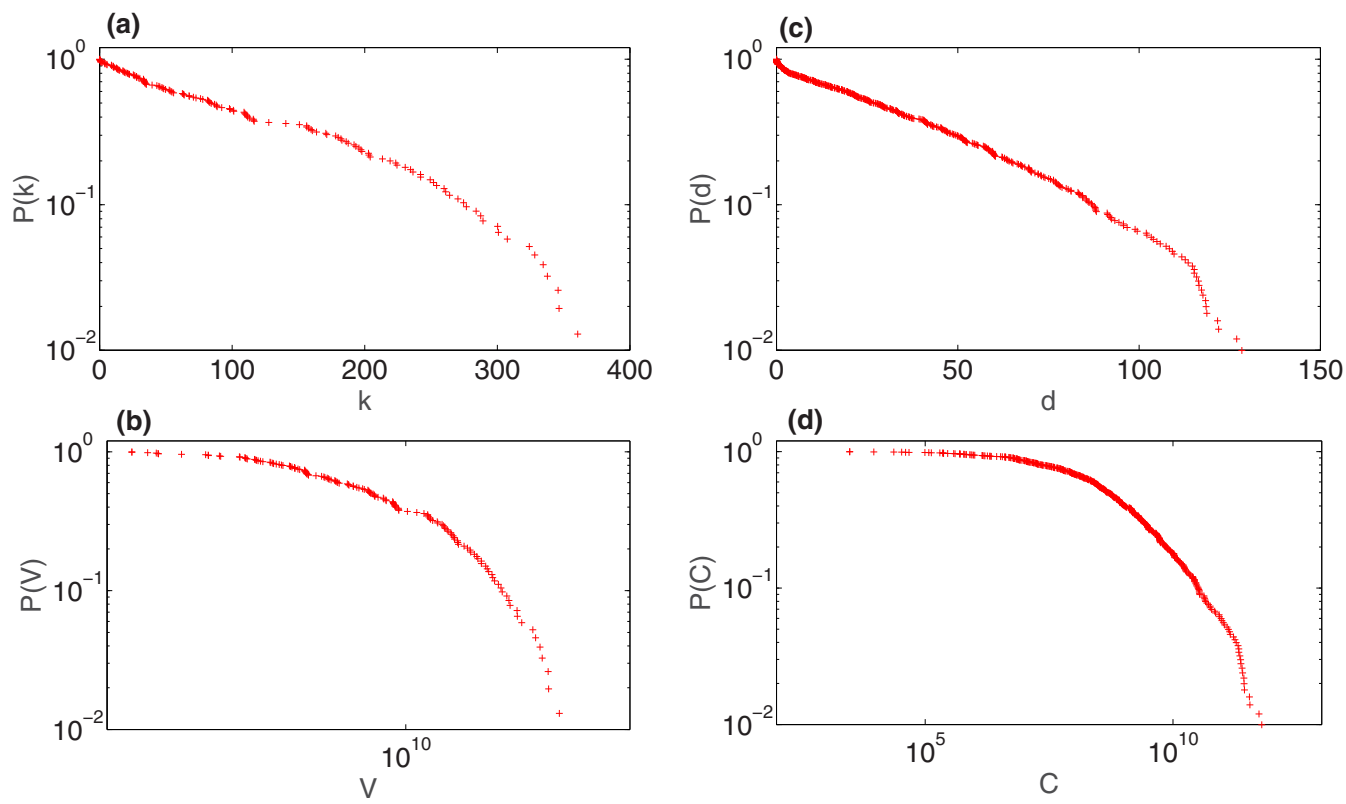

FIG. 1. Empirical cumulative distributions for the degrees of holders $k$ (a) and of issuers $d$ (c), and of the strengths of holders $V$ (b) and of issuers $C$ (d), in the SHS dataset.

the analysis does not contain any explicit reference to any specific country. Also, for the sake of readability, in the following we report results only for the most recent quarter of the dataset (2015Q2), yet our findings are robust across all 24 quarterly snapshots.

\section{RESULTS}

We start by looking at the size of individual exposures between holders and issuers, i.e., the weights of the existing links in the network. As already mentioned, ECAPM weight expectations coincide with those of the original CAPM and MECAPM defined in Eq. (4). If we compare such expectations with the real observed weights (Fig. 2), we see that all reconstruction methods perform well in reproducing the nonzero weights of the network. However, weight uncertainties differ significantly between ECAPM and MECAPM, as shown by the ratio of standard deviations within the ensemble (see

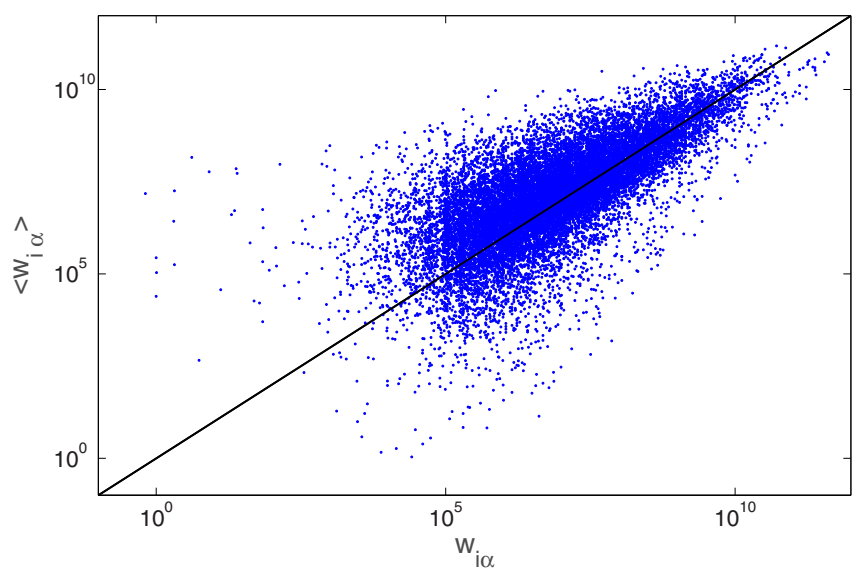

FIG. 2. Comparison between observed weights in the SHS network and CAPM-predicted ones. The black line denotes the identity. the Appendix):

$$
r_{w_{i \alpha}}=\frac{\sigma_{w_{i \alpha}}^{\mathrm{ECAPM}}}{\sigma_{w_{i \alpha}}^{\mathrm{MECAPM}}} \simeq \sqrt{\frac{1}{p_{i \alpha}}-1} .
$$

Since $90 \%$ of the $r_{w_{i \alpha}}$ coefficients are smaller than 1 in our dataset, we can assert that ECAPM is more accurate than MECAPM. Notice the key role played by the topology in lowering the uncertainty of weights estimation: $r_{w_{i \alpha}}<1$ is equivalent to $p_{i \alpha}>1 / 2$. The fact that such a feature holds for country-sector holdings is also consistent with our fitness model ansatz on the relationship between node degrees and strengths.

Next, we perform a key test for the fitness ansatz of our model (Fig. 3), showing that the strength-induced degrees $\left\{\left\langle k_{i}\right\rangle_{\Omega}\right\}_{i=1}^{N}$ and $\left\{\left\langle d_{\alpha}\right\rangle_{\Omega}\right\}_{\alpha=1}^{M}$ predicted by ECAPM interpolate well the empirical values $\left\{k_{i}\left(G_{0}\right)\right\}_{i=1}^{N}$ and $\left\{d_{\alpha}\left(G_{0}\right)\right\}_{\alpha=1}^{M}$, while MECAPM fails to a large extent. This result supports the use of connection probabilities defined by ECAPM as Eq. (3). Then, to further assess the effectiveness of our reconstruction method, we consider three different families of indicators: topological, statistical, and financial ones.

Topological indicators aim at assessing whether a given reconstruction method can effectively reproduce higher-order features which are commonly regarded as most significant for describing a network. Here we focus on the average nearestneighbor degrees $k^{n n}$ and $d^{n n}$ and strengths $V^{n n}$ and $C^{n n}$ [53], namely, the arithmetic mean of degrees and strengths of node neighbors, respectively (see the Appendix). In the context of SHS data, $k^{n n}\left(V^{n n}\right)$ indicate how many exposures (the total portfolio weight) holders have on average for a specific issuer, while $d^{n n}\left(C^{n n}\right)$ indicate how many investors (the total amount of money invested) issuers have on average for a specific holder. As Fig. 4 shows, our method correctly reproduces the observed decreasing trends, interpolating the clouds of points describing the real network. Again, the MECAPM predicts flat 

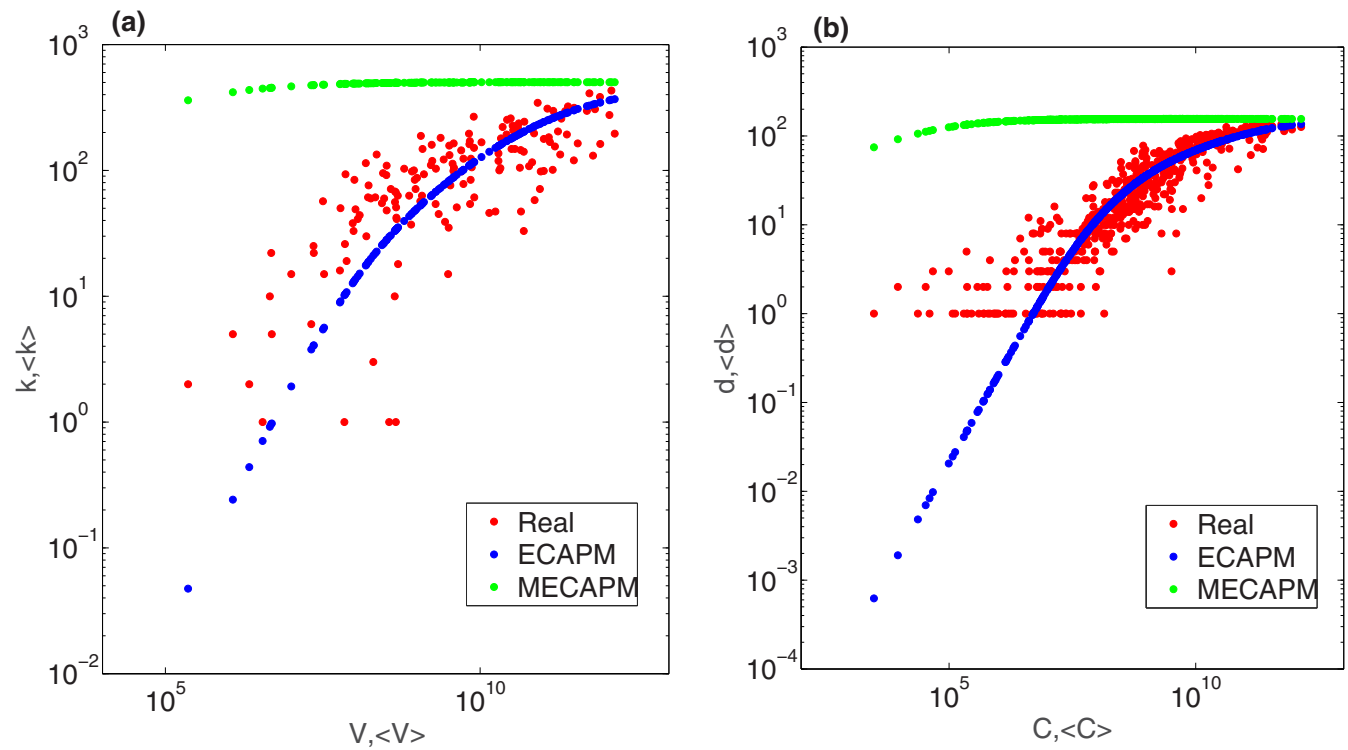

FIG. 3. Empirical relation between strengths and degrees in the data (red points), superimposed with the prediction by ECAPM (blue points) and MECAPM (green points), for both the layers of holders (a) and of issuers (b).

trends which are not compatible with the observed patterns, by systematically over-/underestimating them.

Statistical indicators instead provide information on the details of the reconstructed network structure. More specifically, they quantify the ability to correctly predict the presence of individual links (i.e., the position of ones in the binary biadjacency matrix) and their absence (i.e., the position of zeros). As explained in detail in the Appendix, this is measured by the performance of a binary classifier, thus using the usual percentages of true positives, true negatives, false positives, and false negatives. Table II reports four more informative combinations of such indices: true positive rate (TPR), specificity (SPC), positive predicted value (PPV), and accuracy (ACC). Note that while these numbers refer to $2015 Q 2$, they are remarkably stable across all 24 quarters of our dataset (Fig. 5). In order to correctly interpret the results provided in the table, we recall that while our ECAPM correctly replicates the observed link density $c=\frac{L}{N \cdot M} \simeq 0.24$ ( since $\langle L\rangle_{\Omega}=L$ ), MECAPM returns a density of $c_{\text {MECAPM }} \simeq$ 0.98 . As a trivial consequence, the MECAPM correctly places
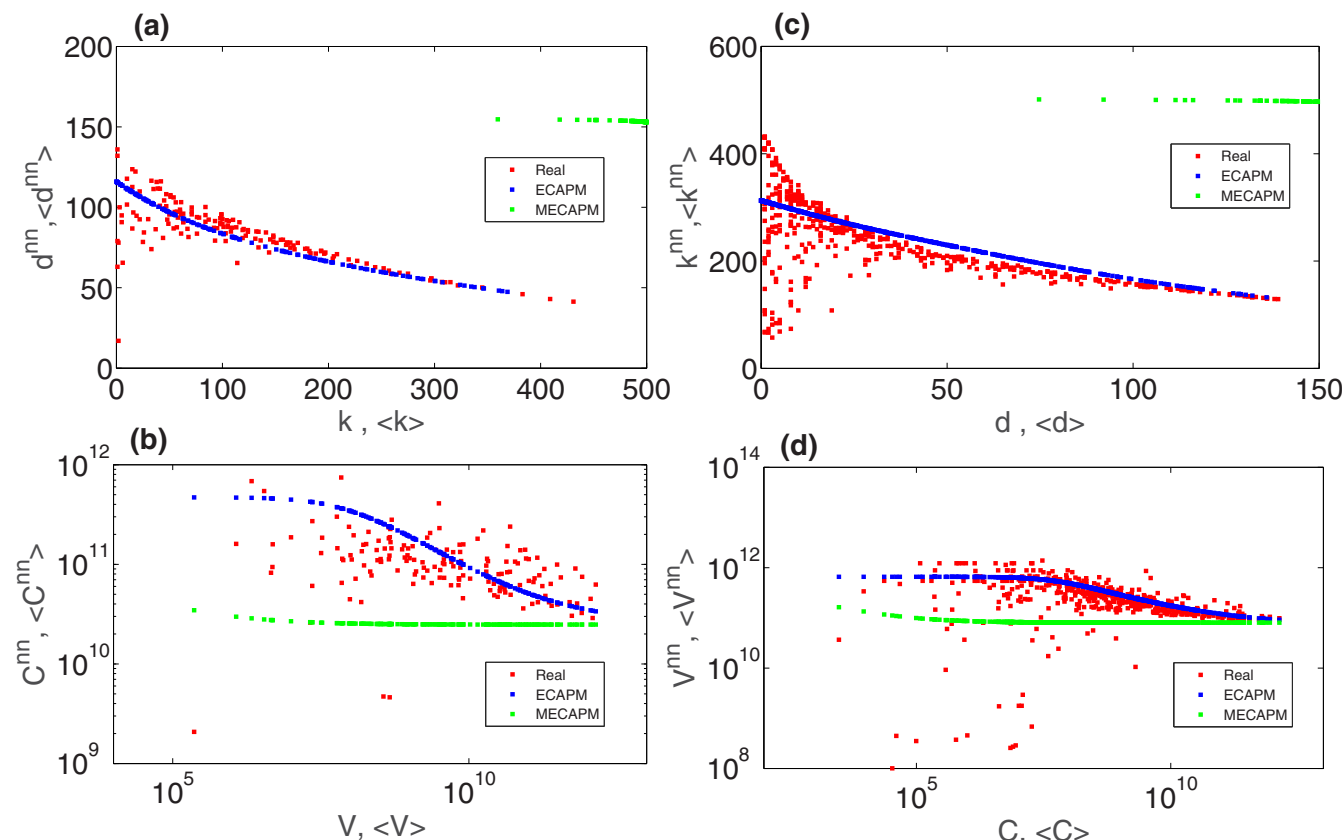

FIG. 4. Empirical values for the average nearest-neighbor degrees $d^{n n}$ vs $k$ (a) and $k^{n n}$ vs $d$ (c), and the average nearest-neighbor strengths $C^{n n}$ vs $V$ (b) and $V^{n n}$ vs $C$ (d) in the data (red points), superimposed with the prediction by ECAPM (blue points) and MECAPM (green points). 
TABLE II. Values of statistical indicators quantifying the performance of ECAPM and MECAPM in reproducing the details of the SHS network structure.

\begin{tabular}{lcc}
\hline \hline & ECAPM & MECAPM \\
\hline True positive rate (TPR) & 0.63 & 0.99 \\
Specificity (SPC) & 0.89 & 0.02 \\
Positive predicted value (PPV) & 0.63 & 0.24 \\
Accuracy (ACC) & 0.83 & 0.25 \\
\hline \hline
\end{tabular}

almost all the ones, obtaining a very high true positive rate $(\mathrm{TPR}=0.99)$. However, this feature also represents a major drawback. In fact, the percentage of correctly recovered zeros is very low ( $\mathrm{SPC}=0.02$ ), pointing out a strong bias towards false positives (the percentage of which amounts to FPR = $1-\mathrm{SPC}=0.98)$. Using the receiver operating characteristic (ROC) curve, a plot which illustrates the performance of a binary classifier as a point with coordinates (TPR,FPR) inside a unit square-the better the classifier, the closer its point to the $(0,1)$ vertex-the point corresponding to MECAPM lies at the top right corner, close to the identity line: this, in turn, implies that its predictive power (PPV) equals that of a random classifier based on the bipartite random graph model (defined by the "homogeneous" assignments $\left.p_{i \alpha} \equiv p=\frac{L}{N \cdot M} \forall i, \alpha\right)$. Thus the MECAPM performance in reproducing the SHS network structure is low, as confirmed by its small overall accuracy. In contrast, ECAPM performs much more accurately, correctly recovering $63 \%$ of the ones and $90 \%$ of zeros and thus achieving a much higher predictive power.

Lastly, financial indicators provide information on how systemic risk is estimated in the reconstructed ensemble. Since we are in the context of bipartite networks of portfolio holdings, here we focus on the risk stemming from sales of illiquid assets and consequent losses during fire sales. In particular, we follow [33] and use the systemicness index $S_{i}$ as a measure of the impact of country sector $i$ on the whole system:

$$
S_{i}=\frac{\Gamma_{i} V_{i}}{E} B_{i} r_{i},
$$

where $\Gamma_{i}=\sum_{j \alpha}\left(w_{j \alpha} l_{\alpha} w_{i \alpha}\right)$ is computed from the illiquidityweighted projection of the bipartite network, $l_{\alpha}$ is the illiquidity parameter of $\alpha, B_{i}$ and $r_{i}$ are the leverage and portfolio return of country sector $i$, and $E$ is the total equity in the system. Note that systemicness as defined by Eq. (7) is at the basis of more recent and refined risk measures [34,57,58], which fully implement dynamical downward spirals of asset prices in the system. Here, since our focus is on network reconstruction and not on systemic risk modeling, we refrain from using such metrics which are more difficult to handle. Yet, Eq. (7) contains quantities which are not accessible through the SHS data. To get rid of them, we can assume homogeneous shocks in the system, as well as equal illiquidity for the asset classes, in order to define the relative systemicness:

$$
\frac{\tilde{S}_{i}}{S_{i}\left(G_{0}\right)}=\frac{\sum_{j \alpha} \tilde{w}_{j \alpha} \tilde{w}_{i \alpha}}{\sum_{j \alpha} w_{j \alpha} w_{i \alpha}} .
$$

We can then use this quantity to test the reconstruction procedures. After some algebra (reported in the Appendix), we find that ECAPM and MECAPM lead to the same predictions in terms of ensemble average for the systemicness. This is a natural consequence of weights expectations which coincide in the two algorithms. However, when single instances of networks drawn from the corresponding reconstructed ensembles are considered, the two methods again differ. Figure 6 shows the relative systemicness values scattered versus the holdings of country sectors. As for the reconstruction of individual weights, the estimates provided by the two methods coincide for the largest nodes. However, MECAPM tends to overestimate the systemicness of small nodes. More importantly, the two methods perform quite differently in estimating the standard deviation of systemicness values. In particular, the standard deviations of the values shown in Fig. 6 are $\varsigma^{\mathrm{MECAPM}} \simeq 2 \times 10^{4}$ and $\varsigma^{\mathrm{ECAPM}} \simeq 10$, and the ratio of the standard deviations within the ensemble, (a)

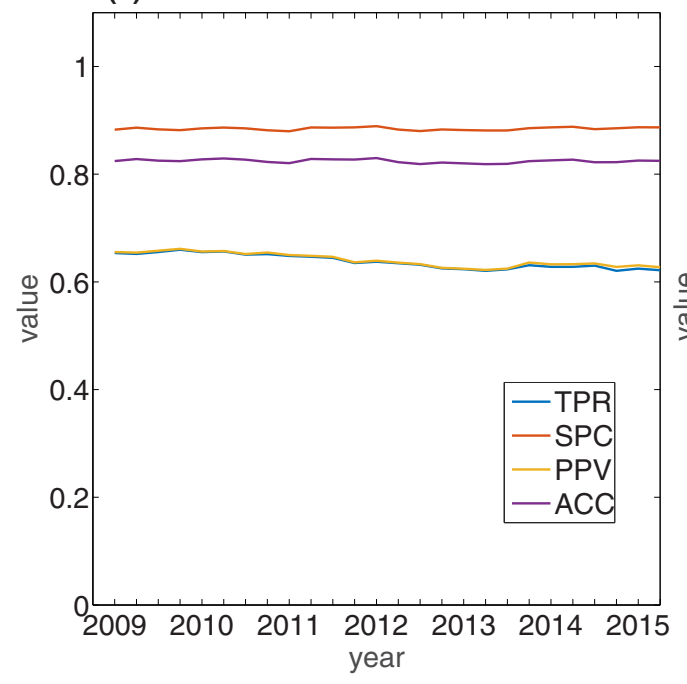

(b)

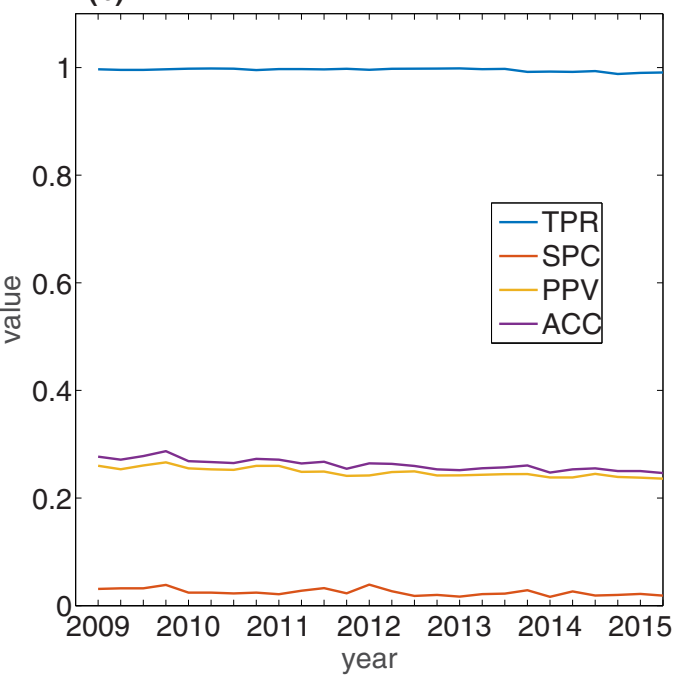

FIG. 5. Performance of ECAPM (a) and MECAPM (b) measured by statistical indicators across the time span of the SHS dataset. 


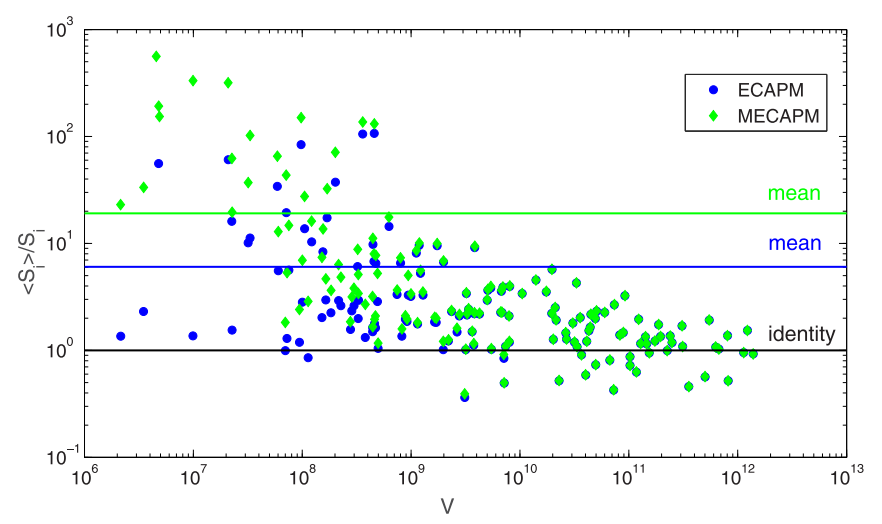

FIG. 6. Relative systemicness index $\tilde{S}_{i} / S_{i}\left(G_{0}\right)$ of the various country sectors as estimated by ECAPM and MECAPM for a particular configuration drawn from the corresponding ensembles. Averages are shown for both methods as horizontal solid lines.

$r_{S_{i}}=\sigma_{S_{i}}^{\mathrm{ECAPM}} / \sigma_{S_{i}}^{\mathrm{MECAPM}}$, is smaller than 1 for $90 \%$ of the nodes in the network. This is particularly relevant in potential stress test applications, since the range of variability of the index within the reconstructed ensemble basically sets the scenarios to be considered: having a small variability is desirable to focus on the most plausible network configurations.

\section{DISCUSSION}

Reconstructing the properties of a network when only partial information is available represents a key open problem in the field of complex systems. Our proposed solution points out that the accuracy of a reconstruction method crucially depends on its ability to reproduce the network topology (i.e., the presence of single links). In fact, without enforcing any topological constraints (e.g., whenever only the strength sequence is enforced), the number of connections is usually overestimated, causing an excess of false positives and, in turn, reducing the predictive power of the reconstruction method. Indeed, a method constraining only nodes strengths assigns the same probability to all configurations with the same strength sequence: since, as pointed out in previous contributions [28,48], the number of configurations satisfying these constraints is usually extremely large, the accuracy of the achieved reconstruction is undermined.

The fundamental role played by topology clearly emerges in the calculation of the errors affecting the estimates of quantities considered. In our case, the standard deviations both for the weights and of the systemicness index are defined in terms of linking probabilities. Remarkably, lower errors are obtained in correspondence of higher probabilities: according to our assumption, this implies that bigger nodes establish more connections, which are in turn less affected by errors. This is relevant especially in terms of systemic risk: bigger nodes are also the most dangerous ones in case of severe losses or default. However, although errors are sensitive to topological details, this is much less the case for the systemicness index measure. $S$, in fact, ignores the node-specific patterns of connections, being purely defined in terms of links weights (see the Appendix). As a consequence, any algorithm providing a satisfactory estimate of the latter performs equally well, irrespective of its performance in reproducing the purely structural quantities (as the presence of the links themselves). In our view, this can represent a drawback of the $S$ index to measure systemic risk. Other measures, on the other hand, are sensitive to topology [32,59]. In particular, in [32] it is shown that the probability of contagion in stock markets strongly depends on connectivity, achieving the minimum value for both the empty and the fully connected configurations and the maximum value for intermediate values of the link density (while the extent of the contagion is a monotonic function of the density). In [59], on the other hand, it is pointed out that portfolio heterogeneity affects the extent and the coordination of feedback effects "triggering transition from stationary dynamics of price returns to non-stationary ones characterized by a steep growth (bubbles) and plunges (bursts) of market prices." Indeed, the analysis of historical datasets that also encompass bubbles and crises represents the subject of future work.

By relying on the fitness model ansatz, ECAPM defines a null model for bipartite weighted networks where strengths replace degree constraints. When available, degrees can be directly used to define the topology using the full BiCM approach [53]. If this is not the case, the applicability of the method strongly depends on the accuracy of the assumed functional relationship between strengths and degrees. While the validity of the fitness ansatz can be assessed by explicitly comparing nodes strengths and degrees (whenever available), its rationale rests upon a simple argument, corroborated by the analysis of other economic and financial networks $[27,28,47,55,56]$ : the higher the importance of a node, the larger we expect its degree to be. Indeed, our method can be applied to any weighted bipartite network for which this argument applies. Similarly, the factorization of any graph probability into the product of single link probabilities characterizing the ECAPM model could be perceived as inherently inadequate to reproduce the real network patterns. Although this may indeed be the cause of the residual deviations between the actual network values and the reconstructed ones, we emphasize that the independence of link probabilities is not postulated by us at any stage: rather, it emerges naturally from the enforcement of purely local constraints (be they degrees or strengths). Put differently, the unbiased solution to the inference problem prescribes to consider independent probabilities. Paradoxically, introducing (more realistic) dependencies would result in a biased inference, if starting from the same observational constraints.

\section{ACKNOWLEDGMENTS}

We thank S. Battiston for useful discussions. This work was supported by the European Union projects MULTIPLEX (Grant No. 317532), DOLFINS (Grant No. 640772), CoeGSS (Grant No. 676547), Shakermaker (Grant No. 687941), and SoBigData (Grant No. 654024). D.G. acknowledges support from the Econophysics foundation (Stichting Econophysics, Leiden, the Netherlands).

G. Caldarelli, G. Cimini, D. Garlaschelli, and T. Squartini developed the method. A. Almog, G. Cimini, and T. Squartini designed and performed the analysis. I. van Lelyveld provided the data set. G. Cimini and T. Squartini wrote the paper. All authors reviewed and approved the paper. 


\section{APPENDIX}

\section{The MECAPM method}

MECAPM combines the constrained entropymaximization technique and the single weights estimation $w_{i \alpha}$ from the CAPM model [30], which reads

$$
\left\langle w_{i \alpha}\right\rangle_{\mathrm{CAPM}}=\frac{V_{i} C_{\alpha}}{W} \quad \forall(i, \alpha) .
$$

This is achieved using the exponential random graph theoretical framework $[44,45]$. Indeed, the CAPM model can be understood as prescribing to constrain the whole set of weights of a given weighted undirected bipartite network $G_{0}$. In this particular case, the probability distribution over the ensemble factorizes into the product of pair-specific geometric probability distributions:

$$
P\left(G_{0}\right)=\prod_{i} \prod_{\alpha} q_{i \alpha}^{w_{i \alpha}}\left(1-q_{i \alpha}\right) .
$$

The generic weight $w_{i \alpha}$ is interpreted as the sum of unitary links, each one behaving as an independent Bernoulli random variable. Each pair-specific probability distribution is thus characterized by a quantity $q_{i \alpha}$, describing the probability that nodes $i$ and $\alpha$ are connected.

The $N \cdot M$ unknown parameters $q_{i \alpha}(i=1 \ldots N, \alpha=1 \ldots M)$ are estimated by exploiting the likelihood conditions [45]

$$
\left\langle w_{i \alpha}\right\rangle_{\mathrm{CAPM}}=\frac{q_{i \alpha}}{1-q_{i \alpha}} \quad \forall(i, \alpha) ;
$$

as a result, one obtains

$$
q_{i \alpha}=\frac{\left\langle w_{i \alpha}\right\rangle_{\mathrm{CAPM}}}{1+\left\langle w_{i \alpha}\right\rangle_{\mathrm{CAPM}}}=\frac{V_{i} C_{\alpha} / W}{1+V_{i} C_{\alpha} / W} \quad \forall(i, \alpha) .
$$

Note that the probability coefficients described by Eq. (A4) solely depend on the (estimated) weights magnitude: as a consequence, the larger the value $\left\langle w_{i \alpha}\right\rangle$, the larger the probability that nodes $i$ and $\alpha$ are connected. Since weights are typically large, the MECAPM method is likely to predict very dense configurations. For what concerns the SHS dataset considered in the present paper, although link probabilities range in the interval $5 \times 10^{-5}-1$, their distribution is characterized by an average value of $\bar{q} \simeq 0.98$ and a standard deviation of $\sigma_{q} \simeq 0.091$. Their variation coefficient is $\sigma_{q} / \bar{q} \simeq 0.093$, indicating that the average faithfully represents the distribution of values (which, practically, coincide with a delta peaked at 1).

\section{The ECAPM method}

Differently from MECAPM, the ECAPM algorithm features a correction term $1 / z$ that accounts for the overall network link density. For the SHS dataset, probability coefficients computed by ECAPM range in the interval $10^{-12}-1$, their average value is $\bar{p} \simeq 0.24$ (coinciding with the network density of links), and their standard deviation is $\sigma_{p} \simeq 0.057$. Their variation coefficient is $\sigma_{p} / \bar{p} \simeq 0.24$, indicating that the average is not representative of the distribution of values, which is thus rather spread over the corresponding support.

The key ingredient of ECAPM is to quantify the tendency of any two nodes of establishing a connection, by assuming the former to depend on the nodes fitness and on the (observed) total number of links. The way in which the ECAPM method works can be better illustrated by considering two special cases which are analytically tractable.

\section{a. Sparse networks}

When considering low-density networks, the probability coefficients can be assumed to be "small." Let us, thus, assume that the Taylor expansion (around $z_{0}=0$ ) of any coefficient can be truncated to the first order:

$$
\begin{aligned}
p_{i \alpha}=\frac{z V_{i} C_{\alpha}}{1+z V_{i} C_{\alpha}} & =z V_{i} C_{\alpha}-\left(z V_{i} C_{\alpha}\right)^{2}+\left(z V_{i} C_{\alpha}\right)^{3} \ldots \\
& \simeq z V_{i} C_{\alpha} .
\end{aligned}
$$

In this case, $z$ can be estimated by imposing $\langle L\rangle=$ $\sum_{i} \sum_{\alpha} p_{i \alpha}=L$, which leads to $z=L / W^{2}$, further implying $p_{i \alpha}=L\left(V_{i} C_{\alpha} / W^{2}\right)$. The latter expression highlights that any two nodes are connected with a probability that is a fraction of the total number of links, properly normalized by the percentage of the total weight represented by the two involved nodes strengths. Analogously, when calculating quantities like nodes degrees, one finds that $\left\langle k_{i}\right\rangle=\sum_{\alpha} p_{i \alpha}=V_{i}(L / W)$ and $\left\langle d_{\alpha}\right\rangle=\sum_{i} p_{i \alpha}=C_{\alpha}(L / W)$.

\section{b. The continuous approximation}

In the sparse case, the empirical distributions of strengths are directly proportional to the empirical distributions of degrees. As a consequence, a scale-free distribution of the former induces a scale-free distribution of the latter. Such an evidence can be used to gain insight into ECAPM also in the general case: upon assuming nodes fitnesses (i.e., nodes strengths) to be drawn from well-defined probability distributions, $V \sim \pi(V)$ and $C \sim \rho(C)$, it is enough to replace each sum with an integral over the aforementioned distributions. As an example, the formula for computing the degree of portfolio $i,\left\langle k_{i}\right\rangle=\sum_{\alpha} p_{i \alpha}$, becomes

$$
\left\langle k_{i}\right\rangle(z, V)=M \int_{\mathcal{C}}\left(\frac{z V_{i} C}{1+z V_{i} C}\right) \rho(C) d C
$$

with $\mathcal{C}$ representing the support of the distribution. By inserting the Taylor expansion in Eq. (A5) into Eq. (A6) we get

$$
\begin{aligned}
\left\langle k_{i}\right\rangle\left(z, V_{i}\right) & =M \int_{\mathcal{C}}\left(\frac{z V_{i} C}{1+z V_{i} C}\right) \rho(C) d C \\
& =M \int_{\mathcal{C}}\left[z V_{i} C-\left(z V_{i} C\right)^{2}+\left(z V_{i} C\right)^{3} \ldots\right] \rho(C) d C \\
& =M\left[z V_{i} \mu_{1}-\left(z V_{i}\right)^{2} \mu_{2}+\left(z V_{i}\right)^{3} \mu_{3} \ldots\right], \quad \text { (A7) }
\end{aligned}
$$

offering a recipe for calculating the numerical value of the statistics of interest, via the computation of the (raw) moments of the involved distributions. As a last comment, note that the formulas for the sparse case are obtained by retaining only the first term in Eq. (A7), and requiring that the first moment of $\rho(C)$ can be estimated by invoking the likelihoodmaximization principle, i.e., $\mu=\bar{C}=\left(\sum_{\alpha} C_{\alpha}\right) / M=W / M$. 


\section{Weights estimation}

As stated in the main text, the ensemble averages of weights under the MECAPM and ECAPM coincide. However, the corresponding statistical fluctuations differ:

$$
\begin{gathered}
\sigma_{w_{i \alpha}}^{2, \mathrm{ECAPM}}=\left\langle w_{i \alpha}\right\rangle_{\mathrm{CAPM}}^{2}\left[\frac{1}{p_{i \alpha}}-1\right], \\
\sigma_{w_{i \alpha}}^{2, \mathrm{MECAPM}}=\left\langle w_{i \alpha}\right\rangle_{\mathrm{CAPM}}\left(1+\left\langle w_{i \alpha}\right\rangle_{\mathrm{CAPM}}\right) .
\end{gathered}
$$

This implies

$$
\begin{aligned}
r_{w_{i \alpha}} & =\frac{\sigma_{w_{i \alpha}}^{\mathrm{ECAPM}}}{\sigma_{w_{i \alpha}}^{\mathrm{MECAPM}}} \\
& =\frac{\left\langle w_{i \alpha}\right\rangle_{\mathrm{CAPM}}^{2}}{\left\langle w_{i \alpha}\right\rangle_{\mathrm{CAPM}}\left(1+\left\langle w_{i \alpha}\right\rangle_{\mathrm{CAPM}}\right)} \sqrt{\frac{1}{p_{i \alpha}}-1} \\
& \simeq \sqrt{\frac{1}{p_{i \alpha}}-1},
\end{aligned}
$$

a ratio that is (strictly) smaller than 1 whenever $p_{i \alpha}>1 / 2$.

\section{Testing the reconstruction methods}

In order to test the performance of both ECAPM and MECAPM in reconstructing the network, we have considered topological, statistical, and financial indicators.

\section{a. Topological indicators}

The first family of indicators consists of quantities providing information on the global, structural organization of a given network. For what concerns the network binary structure, we have considered (i) the degree of, respectively, holders and issuers, $k_{i}(\mathbf{A})=\sum_{\alpha=1}^{M} a_{i \alpha}$ and $d_{\alpha}(\mathbf{A})=\sum_{i=1}^{N} a_{i \alpha}$, and (ii) the average nearest-neighbor degree of holders and issuers [53]:

$$
d_{i}^{n n}(\mathbf{A})=\frac{\sum_{\alpha=1}^{M} a_{i \alpha} d_{\alpha}}{k_{i}}, \quad k_{\alpha}^{n n}(\mathbf{A})=\frac{\sum_{i=1}^{N} a_{i \alpha} k_{i}}{d_{\alpha}} .
$$

We compared these values with their expected counterparts, i.e., $\left\langle k_{i}\right\rangle=\sum_{\alpha=1}^{M}\left\langle a_{i \alpha}\right\rangle,\left\langle d_{\alpha}\right\rangle=\sum_{i=1}^{N}\left\langle a_{i \alpha}\right\rangle$ and

$$
\left\langle d_{i}^{n n}\right\rangle=\frac{\sum_{\alpha=1}^{M}\left\langle a_{i \alpha}\right\rangle\left\langle d_{\alpha}\right\rangle}{\left\langle k_{i}\right\rangle},\left\langle k_{\alpha}^{n n}\right\rangle=\frac{\sum_{i=1}^{N}\left\langle a_{i \alpha}\right\rangle\left\langle k_{i}\right\rangle}{\left\langle d_{\alpha}\right\rangle} .
$$

Note that, for ECAPM in the sparse case, it is $\left\langle d_{i}^{n n}\right\rangle \simeq$ $\left(L / W^{2}\right) \sum_{\alpha} C_{\alpha}^{2}$ and $\left\langle k_{\alpha}^{n n}\right\rangle \simeq\left(L / W^{2}\right) \sum_{i} V_{i}^{2}$, i.e., the expected value of the average nearest-neighbor degree of holders and issuers is constant for all nodes belonging to the same layer. The predictions by MECAPM, on the other hand, can be obtained by considering a very dense network, i.e., $\left\langle a_{i \alpha}\right\rangle=$ $q_{i \alpha} \simeq 1$. In this case, we obtain $\left\langle k_{\alpha}^{n n}\right\rangle_{\mathrm{MECAPM}} \simeq M-1$ and $\left\langle d_{i}^{n n}\right\rangle_{\text {MECAPM }} \simeq N-1$.

For what concerns the weighted structure of the network, we have considered the average nearest-neighbor strengths

$$
C_{i}^{n n}(\mathbf{W})=\frac{\sum_{\alpha=1}^{M} a_{i \alpha} C_{\alpha}}{k_{i}}, \quad V_{\alpha}^{n n}(\mathbf{W})=\frac{\sum_{i=1}^{N} a_{i \alpha} V_{i}}{d_{\alpha}}
$$

and compared them with their expected counterparts:

$$
\left\langle C_{i}^{n n}\right\rangle=\frac{\sum_{\alpha=1}^{M}\left\langle a_{i \alpha}\right\rangle C_{\alpha}}{\left\langle k_{i}\right\rangle}, \quad\left\langle V_{\alpha}^{n n}\right\rangle=\frac{\sum_{i=1}^{N}\left\langle a_{i \alpha}\right\rangle V_{i}}{\left\langle d_{\alpha}\right\rangle} .
$$

As for the average nearest-neighbor degrees, in the sparse case ECAPM predicts flat trends for all nodes belonging to the same layer: $\left\langle C_{i}^{n n}\right\rangle \simeq\left(\sum_{\alpha} C_{\alpha}^{2}\right) / W$ and $\left\langle k_{\alpha}^{n n}\right\rangle \simeq\left(\sum_{i} V_{i}^{2}\right) / W$. MECAPM predictions are instead obtained by again considering a very dense network: $\left\langle V_{\alpha}^{n n}\right\rangle_{\text {MECAPM }} \simeq W /(N-1)$, $\left\langle C_{i}^{n n}\right\rangle_{\mathrm{MECAPM}} \simeq W /(M-1)$.

\section{b. Statistical indicators}

Statistical indicators are compactly represented by the socalled confusion matrix, a $4 \times 4$ matrix the entries of which represent the number of true positives, true negatives, false positives, and false negatives. Let us briefly explain why these concepts are useful for our analysis.

Reconstructing a network $G_{0}$ means providing an algorithm to estimate the presence and the weight of the connections. If we limit our analysis to the binary structure only (represented by the binary matrix $\mathbf{A}$, with $a_{i \alpha}=\Theta\left[w_{i \alpha}\right]$ ), this implies "guessing" the topological structure of the network, namely the position of zeros and ones in the matrix. For each entry of the considered biadjacency matrix, four different cases are possible.

(a) $a_{i \alpha}=1$ and we correctly predict $\tilde{a}_{i \alpha}=1$. When this is the case, we have a true positive.

(b) $a_{i \alpha}=1$ but we predict $\tilde{a}_{i \alpha}=0$. In this case, we have a false negative.

(c) $a_{i \alpha}=0$ and we correctly predict $\tilde{a}_{i \alpha}=0$. In this case, we have a true negative.

(d) $a_{i \alpha}=0$ but we predict $\tilde{a}_{i \alpha}=1$. When this is the case, we have a false positive.

Given the observed biadjacency matrix $\mathbf{A}$ and the reconstructed matrix $\tilde{\mathbf{A}}$, we can straightforwardly count the total number of true positives as the pointwise product of the two matrices

$$
\mathrm{TP}=\sum_{i} \sum_{\alpha} a_{i \alpha} \tilde{a}_{i \alpha}
$$

(the generic addendum of which is 1 iff both $a_{i j}=1$ and $\tilde{a}_{i j}=1$ ), the total number of false negatives as

$$
\mathrm{FN}=\sum_{i} \sum_{\alpha} a_{i \alpha}\left(1-\tilde{a}_{i \alpha}\right)=L\left(G_{0}\right)-\mathrm{TP}
$$

[where $L\left(G_{0}\right)$ is total number of observed links], the total number of true negatives as

$$
\begin{aligned}
\mathrm{TN} & =\sum_{i} \sum_{\alpha}\left(1-a_{i \alpha}\right)\left(1-\tilde{a}_{i \alpha}\right) \\
& =N \cdot M-\tilde{L}-L\left(G_{0}\right)+\mathrm{TP}
\end{aligned}
$$

(the generic addendum of which is 1 iff both $a_{i j}=0$ and $\tilde{a}_{i j}=0$ ), and the total number of false positives as

$$
\begin{aligned}
\mathrm{FP} & =\sum_{i} \sum_{\alpha}\left(1-a_{i \alpha}\right) \tilde{a}_{i \alpha} \\
& =\tilde{L}-\mathrm{TP}=\left[N \cdot M-L\left(G_{0}\right)\right]-\mathrm{TN}
\end{aligned}
$$

the first addendum of which is the number of zeros in the real matrix A. 
The information provided by TP, FN, TN, and FP is usually given by combinations of them. Sensitivity (or true positive rate) is defined as

$$
\mathrm{TPR}=\frac{\mathrm{TP}}{\mathrm{TP}+\mathrm{FN}}=\frac{\mathrm{TP}}{L\left(G_{0}\right)}
$$

and quantifies the percentage of ones that are correctly recovered by a reconstruction method. Specificity (or true negative rate) is defined as

$$
\mathrm{SPC}=\frac{\mathrm{TN}}{\mathrm{FP}+\mathrm{TN}}=\frac{\mathrm{TN}}{N \cdot M-L\left(G_{0}\right)}
$$

and quantifies the percentage of zeros that are correctly recovered by a reconstruction method. The false positive rate, defined as FPR $=1-\mathrm{SPC}$, is usually compared to TPR in order to evaluate the performance of a given algorithm with respect to a random classifier. In fact, whenever TPR and FPR coincide, the performance of the considered model equals that of a random classifier (in other words, the model representative point lies on the identity line of the ROC curve). Precision (or positive predicted value) is defined as

$$
\mathrm{PPV}=\frac{\mathrm{TP}}{\mathrm{TP}+\mathrm{FP}}
$$

and measures the performance of an algorithm in correctly placing the ones with respect to the total number of predicted ones. Finally, accuracy is

$$
\mathrm{ACC}=\frac{\mathrm{TP}+\mathrm{TN}}{\mathrm{TP}+\mathrm{TN}+\mathrm{FP}+\mathrm{FN}}=\frac{\mathrm{TP}+\mathrm{TN}}{N \cdot M}
$$

and measures the overall performance of a reconstruction method in correctly placing both ones and zeros.

Since both the ECAPM and the MECAPM methods deal with an entire ensemble of candidate matrices $\tilde{\mathbf{A}}$, we are interested in estimating the expected values of the aforementioned indices. Their ensemble averages are reported in Table III. For the sake of illustration, let us explicitly derive them for the ECAPM method in the sparse regime and for the MECAPM method, below.
By resting upon the continuous approximation, the expected number of true positives for ECAPM is

$$
\begin{aligned}
\langle\mathrm{TP}\rangle & =L\left(G_{0}\right) \int_{\mathcal{V}} \int_{\mathcal{C}} p(V, C) \rho(C) \pi(V) d C d V \\
& \simeq L\left(G_{0}\right) \int_{\mathcal{V}} \int_{\mathcal{C}}(z V C) \rho(C) \pi(V) d C d V \\
& =L\left(G_{0}\right) z \lambda \mu=L\left(G_{0}\right)^{2} /(N \cdot M),
\end{aligned}
$$

where we have truncated the analytical expression of $p(V, C)$ at the first order and estimated the first raw moment of the two distributions as $\lambda=\bar{V}=W / N$ and $\mu=\bar{C}=W / M$. In the same regime of Eq. (A23), $\langle\mathrm{TPR}\rangle=L\left(G_{0}\right) /(N \cdot M)$, i.e., the expected sensitivity coincides with the link density.

In the case of MECAPM, instead, TPR and FPR practically coincide (see Table II). This implies

$$
\begin{aligned}
\langle\mathrm{TPR}\rangle & =1-\langle\mathrm{SPC}\rangle \\
\Rightarrow \frac{\langle\mathrm{TP}\rangle}{L_{\text {true }}} & =\frac{\langle L\rangle-\langle\mathrm{TP}\rangle}{N \cdot M-L_{\text {true }}} \\
\Rightarrow \frac{\langle\mathrm{TP}\rangle}{\langle L\rangle} & =\langle\mathrm{PPV}\rangle=\frac{L\left(G_{0}\right)}{N \cdot M},
\end{aligned}
$$

i.e., the MECAPM predictive power coincides with the network link density, which is the PPV of the random graph model.

Interestingly, the performance of the ECAPM method in the sparse case and that of MECAPM in recovering the correct number of ones are very similar (as confirmed by their close PPV values). However, in the regime of Eq. (A23), the expected accuracy for ECAPM reads $\langle$ ACC $\rangle \simeq$ $1+2 L\left(G_{0}\right) /(N \cdot M)$, which can be quite large if the network is very sparse. In other words, the overall accuracy of ECAPM in reconstructing sparse networks can still be large, the reason lying in the large number of zeros correctly recovered.

\section{c. Financial indicators}

The third family of indicators aims at quantifying the systemicness of nodes belonging to a financial network. We follow [33] and adopt the systemicness index $S_{i}$ :

$$
S_{i}=\frac{\Gamma_{i} V_{i}}{E} B_{i} r_{i}
$$

TABLE III. Statistical indicators used to evaluate the performance of ECAPM and MECAPM in reproducing the observed network structure.

\begin{tabular}{ll}
\hline \hline $\mathrm{ECAPM}$ & $\mathrm{MECAPM}$ \\
\hline$\langle\mathrm{TP}\rangle_{\mathrm{ECAPM}}=\sum_{i} \sum_{\alpha} a_{i \alpha} p_{i \alpha}$ & $\langle\mathrm{TP}\rangle_{\mathrm{MECAPM}} \simeq L\left(G_{0}\right)$ \\
$\langle\mathrm{TN}\rangle_{\mathrm{ECAPM}}=\sum_{i} \sum_{\alpha}\left(1-a_{i \alpha}\right)\left(1-p_{i \alpha}\right)=N \cdot M-2 L\left(G_{0}\right)+\langle\mathrm{TP}\rangle$ & $\langle\mathrm{TN}\rangle_{\mathrm{MECAPM}} \simeq 0$ \\
$\langle\mathrm{FP}\rangle_{\mathrm{ECAPM}}=\left[N \cdot M-L\left(G_{0}\right)\right]-\langle\mathrm{TN}\rangle_{\mathrm{ECAPM}}=L\left(G_{0}\right)-\langle\mathrm{TP}\rangle_{\mathrm{ECAPM}}$ & $\langle\mathrm{FP}\rangle_{\mathrm{MECAPM}} \simeq N \cdot M-L\left(G_{0}\right)$ \\
$\langle\mathrm{FN}\rangle_{\mathrm{ECAPM}}=L\left(G_{0}\right)-\langle\mathrm{TP}\rangle_{\mathrm{ECAPM}}$ & $\langle\mathrm{FN}\rangle_{\mathrm{MECAPM}} \simeq 0$ \\
$\langle\mathrm{TPR}\rangle_{\mathrm{ECAPM}}=\langle\mathrm{TP}\rangle_{\mathrm{ECAPM}} / L\left(G_{0}\right)$ & $\langle\mathrm{TPR}\rangle_{\mathrm{MECAPM}} \simeq 1$ \\
$\langle\mathrm{FPR}\rangle_{\mathrm{ECAPM}}=\frac{L\left(G_{0}\right)-\langle\mathrm{TP}\rangle_{\mathrm{ECAPM}}}{N \cdot M-L\left(G_{0}\right)}$ & $\langle\mathrm{FPR}\rangle_{\mathrm{MECAPM}} \simeq 1$ \\
$\langle\mathrm{SPC}\rangle_{\mathrm{ECAPM}}=\frac{\langle\mathrm{TN}\rangle_{\mathrm{ECAPM}}}{N \cdot M-L\left(G_{0}\right)}=1-\langle\mathrm{FPR}\rangle_{\mathrm{ECAPM}}$ & $\langle\mathrm{SPC}\rangle_{\mathrm{MECAPM}} \simeq 0$ \\
$\langle\mathrm{PPV}\rangle_{\mathrm{ECAPM}}=\frac{\langle\mathrm{TP}\rangle_{\mathrm{ECAPM}}}{\langle\mathrm{TP}\rangle_{\mathrm{ECAPM}}+\langle\mathrm{FP}\rangle_{\mathrm{ECAPM}}}=\frac{\langle\mathrm{TP}\rangle_{\mathrm{ECAPM}}}{L\left(G_{0}\right)}$ & $\langle\mathrm{PPV}\rangle_{\mathrm{MECAPM}} \simeq \frac{L\left(G_{0}\right)}{N \cdot M}=c\left(G_{0}\right)$ \\
$\langle\mathrm{ACC}\rangle_{\mathrm{ECAPM}}=\frac{\langle\mathrm{TP}\rangle_{\mathrm{ECAPM}}+\langle\mathrm{TN}\rangle_{\mathrm{ECAPM}}}{N \cdot M}=1-2 c\left(G_{0}\right)+2 \frac{\langle\mathrm{TP}\rangle_{\mathrm{ECAPM}}}{N \cdot M}$ & $\langle\mathrm{ACC}\rangle_{\mathrm{MECAPM}} \simeq c\left(G_{0}\right)$ \\
\hline \hline
\end{tabular}


which can be compared with its expected counterpart $\left\langle S_{i}\right\rangle$ via the ratio $\left\langle S_{i}\right\rangle / S_{i}$. After some algebraic manipulations [30], such a ratio can be rewritten as

$$
\frac{\left\langle S_{i}\right\rangle}{S_{i}}=\frac{\sum_{j} \sum_{\alpha}\left\langle w_{i \alpha} w_{j \alpha}\right\rangle}{\sum_{j} \sum_{\alpha} w_{i \alpha} w_{j \alpha}}=\frac{\sum_{\alpha}\left\langle w_{i \alpha}^{2}\right\rangle+\sum_{j \neq i}\left\langle w_{i \alpha}\right\rangle\left\langle w_{j \alpha}\right\rangle}{\sum_{\alpha} w_{i \alpha} C_{\alpha}} .
$$

For ECAPM, this ratio can be further simplified upon considering that $\left\langle w_{i \alpha}\right\rangle=\frac{V_{i} C_{\alpha}}{W}$ and $\left\langle w_{i \alpha}^{2}\right\rangle=\left(\frac{V_{i} C_{\alpha}}{W p_{i \alpha}}\right)^{2} p_{i \alpha}$, which leads to the expression

$$
\frac{\left\langle S_{i}\right\rangle_{\mathrm{ECAPM}}}{S_{i}}=\frac{\sum_{\alpha}\left(\frac{V_{i} C_{\alpha}}{W p_{i \alpha}}\right)^{2} p_{i \alpha}+\frac{V_{i} C_{\alpha}}{W}\left[C_{\alpha}-\frac{V_{i} C_{\alpha}}{W}\right]}{\sum_{\alpha} w_{i \alpha} C_{\alpha}} .
$$

In the case of MECAPM instead we have $\left\langle w_{i \alpha}\right\rangle_{\text {MECAPM }}=$ $\frac{V_{i} C_{\alpha}}{W}$ and $\left\langle w_{i \alpha}^{2}\right\rangle_{\text {MECAPM }}=\frac{V_{i} C_{\alpha}}{W}\left(1+2 \frac{V_{i} C_{\alpha}}{W}\right)$, which leads to the expression

$$
\frac{\left\langle S_{i}\right\rangle_{\mathrm{MECAPM}}}{S_{i}}=\frac{\sum_{\alpha} \frac{V_{i} C_{\alpha}}{W}\left[1+C_{\alpha}+\frac{V_{i} C_{\alpha}}{W}\right]}{\sum_{\alpha} w_{i \alpha} C_{\alpha}} .
$$

Since, in both cases, the term $\frac{V_{i} C_{\alpha}^{2}}{W}$ dominates over the other ones and $\left\langle w_{i \alpha}\right\rangle_{\mathrm{ECAPM}}=\left\langle w_{i \alpha}\right\rangle_{\mathrm{MECAPM}}=\left\langle w_{i \alpha}\right\rangle_{\mathrm{CAPM}}$, we have

$$
\begin{aligned}
\frac{\left\langle S_{i}\right\rangle_{\mathrm{ECAPM}}}{S_{i}} & \simeq \frac{\left\langle S_{i}\right\rangle_{\mathrm{MECAPM}}}{S_{i}} \simeq \frac{\sum_{\alpha} \frac{V_{i} C_{\alpha}^{2}}{W}}{\sum_{\alpha} w_{i \alpha} C_{\alpha}} \\
& =\frac{\sum_{\alpha}\left\langle w_{i \alpha}\right\rangle_{\mathrm{CAPM}} C_{\alpha}}{\sum_{\alpha} w_{i \alpha} C_{\alpha}} .
\end{aligned}
$$

Equation (A29) implies that, among the reconstruction methods satisfying the strength constraints, those which are expected to better reproduce the systemicness index are the ones better reproducing the observed weights. As discussed in the main text, the prescription $\left\langle w_{i \alpha}\right\rangle_{\mathrm{ECAPM}}=\left\langle w_{i \alpha}\right\rangle_{\mathrm{MECAPM}}=$ $\frac{V_{i} C_{\alpha}}{W}$ is particularly successful in reproducing the largest weights of the SHS network (beside ensuring that $\left\langle C_{\alpha}\right\rangle=C_{\alpha}$ ).

The statistical fluctuations affecting the $S_{i}$ index are

$$
\begin{aligned}
\sigma_{S_{i}}^{2, \mathrm{MECAPM}}= & \sum_{\alpha}\left\langle w_{i \alpha}\right\rangle\left[\left(1+2\left\langle w_{i \alpha}\right\rangle\right) \sum_{j}\left\langle w_{j \alpha}\right\rangle^{2}\right. \\
& \left.+C_{\alpha}\left(1+C_{\alpha}\right)+C_{\alpha}\left\langle w_{i \alpha}\right\rangle\left(2+C_{\alpha}\right)\right], \\
\sigma_{S_{i}}^{2, \mathrm{ECAPM}}= & \sum_{\alpha} \frac{\left\langle w_{i \alpha}\right\rangle^{2}}{p_{i \alpha}}\left[\sum_{j} \frac{\left\langle w_{j \alpha}\right\rangle^{2}}{p_{j \alpha}}\right. \\
& \left.+C_{\alpha}^{2}\left(1-p_{i \alpha}\right)-\sum_{j}\left\langle w_{j \alpha}\right\rangle^{2}\right] .
\end{aligned}
$$

Interestingly enough, plotting the vector of ratios

$$
r_{S_{i}}=\frac{\sigma_{S_{i}}^{\mathrm{ECAPM}}}{\sigma_{S_{i}}^{\mathrm{MECAPM}}}
$$

versus the nodes strengths $V_{i}$ reveals a functional dependency $r_{S_{i}} \propto V_{i}^{-1 / 2}$. Again, this confirms that ECAPM outperforms MECAPM in providing an estimate of the systemicness index $S_{i}$ for the larger institutions, which become less sensitive to statistical fluctuations.
[1] L. Eisenberg and T. H. Noe, Manag. Sci. 47, 236 (2001).

[2] G. Bonanno, G. Caldarelli, F. Lillo, and R. N. Mantegna, Phys. Rev. E 68, 046130 (2003).

[3] P. Gai and S. Kapadia, Proc. R. Soc. A 466, 2401 (2010).

[4] A. G. Haldane and R. M. May, Nature (London) 469, 351 (2011).

[5] D. Acemoglu, A. Ozdaglar, and A. Tahbaz-Salehi, Am. Econ. Rev. 105, 564 (2015).

[6] G. Cimini and M. Serri, PLoS ONE 11, e0161642 (2016).

[7] F. Allen and D. Gale, J. Polit. Econ. 108, 1 (2000).

[8] J. A. Chan-Lau, M. Espinosa, K. Giesecke, and J. A. Solé, Assessing the Systemic Implications of Financial Linkages, Technical Report, IMF Global Financial Stability Report, 2009.

[9] M. K. Brunnermeier, J. Econ. Perspect. 23, 77 (2009).

[10] A. Krause and S. Giansante, J. Econ. Behav. Organ. 83, 583 (2012).

[11] S. Battiston, G. Caldarelli, R. May, T. Roukny, and J. E. Stiglitz, Proc. Natl. Acad. Sci. USA 113, 10031 (2016).

[12] G. Iori, S. Jafarey, and F. G. Padilla, J. Econ. Behav. Organ. 61 , 525 (2006).

[13] H. Elsinger, A. Lehar, and M. Summer, Manag. Sci. 52, 1301 (2006).

[14] E. W. Nier, J. Yang, T. Yorulmazer, and A. Alentorn, J. Econ. Dyn. Control 31, 2033 (2007).

[15] M. Bardoscia, S. Battiston, F. Caccioli, and G. Caldarelli, Nat. Commun. 8, 14416 (2017).
[16] S. Battiston, J. D. Farmer, A. Flache, D. Garlaschelli, A. G. Haldane, H. Heesterbeek, C. Hommes, C. Jaeger, R. May, and M. Scheffer, Science 351, 818 (2016).

[17] S. J. Wells, Financial Interlinkages in the United Kingdom's Interbank Market and the Risk of Contagion, Bank of England Working Paper No. 230, 2004.

[18] C. Upper, J. Finan. Stab. 7, 111 (2011).

[19] P. E. Mistrulli, Assessing Financial Contagion in the Interbank Market: Maximum Entropy versus Observed Interbank Lending Patterns, Temi di discussione (Economic working papers), Bank of Italy Economic Working Paper No. 641, 2007.

[20] I. Mastromatteo, E. Zarinelli, and M. Marsili, J. Stat. Mech. (2012) P03011

[21] P. Baral and J. P. Fique, Estimation of Bilateral Exposures-A Copula Approach (Mimeo, 2012).

[22] M. Drehmann and N. Tarashev, J. Finan. Interm. 22, 586 (2013).

[23] G. Hałaj and C. Kok, Computat. Manag. Sci. 10, 157 (2013).

[24] K. Anand, B. Craig, and G. von Peter, Quantit. Finan. 15, 625 (2015).

[25] M. Montagna and T. Lux, Quantit. Finan. 17, 101 (2017).

[26] T. A. Peltonen, M. Rancan, and P. Sarlin, Interconnectedness of the Banking Sector as a Vulnerability to Crises, ECB, European Central Bank Working Paper No. 1866, 2015.

[27] G. Cimini, T. Squartini, D. Garlaschelli, and A. Gabrielli, Sci. Rep. 5, 15758 (2015). 
[28] G. Cimini, T. Squartini, A. Gabrielli, and D. Garlaschelli, Phys. Rev. E 92, 040802 (2015).

[29] A. Gandy and L. A. M. Veraart, Manag. Sci. (2016), doi:10.1287/mnsc.2016.2546.

[30] D. Di Gangi, F. Lillo, and D. Pirino, arXiv:1509.00607 (2015).

[31] A. Shleifer and R. W. Vishny, J. Econ. Perspect. 25, 29 (2011).

[32] F. Caccioli, M. Shrestha, C. Moore, and J. D. Farmer, J. Bank. Finan. 46, 233 (2014).

[33] R. Greenwood, A. Landier, and D. Thesmar, J. Finan. Econ. 115, 471 (2015).

[34] R. Cont and L. Wagalath, Math. Finan. 26, 835 (2016).

[35] S. Gualdi, G. Cimini, K. Primicerio, R. D. Clemente, and D. Challet, Sci. Rep. 6, 39467 (2016).

[36] T. Adrian and H. S. Shin, J. Finan. Intermed. 19, 418 (2010).

[37] D. W. Diamond and R. G. Rajan, Fear of Fire Sales and the Credit Freeze, National Bureau of Economic Research Working Paper No. 14925, 2009.

[38] J. M. Berrospide, Bank Liquidity Hoarding and the Financial Crisis: An Empirical Evaluation, Finance and Economics Discussion Series No. 03 (Board of Governors of the Federal Reserve System US, 2013).

[39] D. Gale and T. Yorulmazer, Theor. Econ. 8, 291 (2013).

[40] V. V. Acharya and O. Merrouche, Rev. Finan. 17, 107 (2013).

[41] G. Silvia and C.-P. Georg, A Network View on Interbank Market Freezes, Banque de France Working Paper No. 531, 2014.

[42] N. Musmeci, S. Battiston, G. Caldarelli, M. Puliga, and A. Gabrielli, J. Stat. Phys. 151, 720 (2013).

[43] G. Cimini, T. Squartini, N. Musmeci, M. Puliga, A. Gabrielli, D. Garlaschelli, S. Battiston, and G. Caldarelli, Reconstructing topological properties of complex networks using the fitness model, in Proceedings of the Social Informatics: Socinfo 2014 International Workshops (Springer, New York, 2015), pp. 323-333.

[44] J. Park and M. E. J. Newman, Phys. Rev. E 70, 066117 (2004).
[45] T. Squartini and D. Garlaschelli, New J. Phys. 13, 083001 (2011).

[46] G. Caldarelli, A. Capocci, P. De Los Rios, and M. A. Muñoz, Phys. Rev. Lett. 89, 258702 (2002).

[47] D. Garlaschelli and M. I. Loffredo, Phys. Rev. Lett. 93, 188701 (2004).

[48] R. Mastrandrea, T. Squartini, G. Fagiolo, and D. Garlaschelli, New J. Phys. 16, 043022 (2014).

[49] P. Mazzarisi and F. Lillo, Methods for reconstructing interbank networks from limited information: A comparison, in Econophysics and Sociophysics: Recent Progress and Future Directions, edited by F. Abergel, H. Aoyama, B. K. Chakrabarti, A. Chakraborti, N. Deo, D. Raina, and I. Vodenska (Springer, New York, 2017), pp. 201-215.

[50] K. Anand, I. van Lelyveld, A. Banai, T. Christiano Silva, S. Friedrich, R. Garratt, G. Halaj, I. Hansen, B. Howell, H. Lee, S. M. Jaramillo, J. Molina-Borboa, S. Nobili, S. Rajan, S. R. S. de Souza, D. Salakhova, and L. Silvestri, J. Finan. Stab. (2017), doi:10.1016/j.jfs.2017.05.012.

[51] J. Lintner, The Rev. Econ. Stat. 47, 13 (1965).

[52] J. Mossin, Econometrica 34, 768 (1966).

[53] F. Saracco, R. Di Clemente, A. Gabrielli, and T. Squartini, Sci. Rep. 5, 10595 (2015).

[54] T. Squartini, G. Cimini, A. Gabrielli, and D. Garlaschelli, Appl. Netw. Sci. 2, 3 (2017).

[55] D. Garlaschelli, S. Battiston, M. Castri, V. D. Servedio, and G. Caldarelli, Physica A 350, 491 (2005).

[56] G. De Masi, G. Iori, and G. Caldarelli, Phys. Rev. E 74, 066112 (2006).

[57] R. Cont and E. F. Schaanning, Fire Sales, Indirect Contagion and Systemic Stress Testing (2017), doi:10.2139/ssrn.2541114.

[58] M. Getmansky, G. Girardi, K. W. Hanley, S. Nikolova, and L. Pelizzon, Portfolio similarity and asset liquidation in the insurance industry, in Fourth Annual Conference on Financial Market Regulation (2017), doi:10.2139/ssrn.2749691.

[59] F. Corsi, S. Marmi, and F. Lillo, Oper. Res. 64, 1073 (2016). 